\title{
Sampling with Hammersley and Halton Points
}

\author{
Tien-Tsin Wong \\ The Chinese University of Hong Kong \\ Wai-Shing Luk \\ Katholieke Universiteit Leuven \\ Pheng-Ann Heng \\ The Chinese University of Hong Kong
}

\begin{abstract}
The Hammersley and Halton point sets, two well known low discrepancy sequences, have been used for quasi-Monte Carlo integration in previous research. A deterministic formula generates a uniformly distributed and stochastic-looking sampling pattern, at low computational cost. The Halton point set is also useful for incremental sampling. In this paper, we discuss detailed implementation issues and our experience of choosing suitable bases of the point sets, not just on the 2D plane, but also on a spherical surface. The sampling scheme is also applied to ray tracing, with a significant improvement in error.
\end{abstract}

\section{Introduction}

Different sampling techniques are used in computer graphics for the purpose of antialiasing. The two easiest ways to sample are randomly and regularly. Unfortunately, random sampling gives a noisy result. Regular sampling gives aliasing, which requires many extra samples to reduce. Several techniques in between these two have been proposed. The thesis of Shirley [19] surveys the common sampling techniques, including jittered [3], semi-jittered, Poisson disk and N-rooks sampling. Cychosz [6] generated sampling jitters using look-up tables. Chiu et al. [2] combined jittered and $\mathrm{N}$-rooks methods to design a new multi-jittered sampling. Cross [4] used a genetic algorithm to find the optimal sampling pattern for uniformly distributed edges. All these methods make tradeoffs between noisiness and aliasing.

A sampling technique is hierarchical if when it is required to generate $N_{0}$ samples, the result coincides with the first $N_{0}$ samples generated for $N=N_{0}+1$ where $N_{0}$ is a positive integer. This is a useful feature, since the number of samples can be incrementally increased without recalculating the previous ones. Shoemake [20] mentioned a means to incrementally sample the 1D space while keeping the samples as uniform as possible. However, this method is not easy to be generalized to higher 
dimensions. Among previously mentioned methods, only Poisson disk and random sampling are hierarchical.

Discrepancy analysis measures sample point equidistribution, that is, measures how uniformly distributed the point set is. Shirley [18] first applied it to the sampling problem. The possible importance of discrepancy in computer graphics is also pointed out by Niederreiter [14]. Dobkin et al. [7, 9, 8] proposed various methods to measure the discrepancy of sampling patterns and to generate the patterns [8]. Heinrich and Keller [11, 12,13] and Ohbuchi and Aono [16] applied low discrepancy sequences to Monte Carlo integration in radiosity applications.

In this paper, we discusses two useful low discrepancy sequences, namely Hammersley and Halton. They have been used in numerical $[17,23,1]$ and graphics $[11,12,13,16]$ applications, with a significant improvement in terms of error. Previous researches mainly concentrate on sample generation on the 2D plane, cube and hypercube. It has been recently found [5] that mapping Hammersley points with base of 2 to the surface of a sphere also give uniformly distributed directional vectors. We discuss the implementation issues and experience in choosing suitable bases of Hammersley and Halton points on 2D plane and spherical surface.

The mathematical formulation is briefly described in Section 2 . Section 3 compares sampling patterns generated using different bases. Ray tracing experiments to verify the usefulness of the method, are discussed in section 4 . The $\mathrm{C}$ implementations are listed in the appendix.

\section{Hammersley and Halton Points}

We first describe the definition of Hammersley and Halton points, then discuss their implementation in detail. For more mathematical detail, readers are referred to the mathematics literature $[15,5]$.

Each nonnegative integer $k$ can be expanded using a prime base $p$ :

$$
k=a_{0}+a_{1} p+a_{2} p^{2}+\ldots+a_{r} p^{r} .
$$

where each $a_{i}$ is an integer in $[0, p-1]$. Now define a function $\Phi_{p}$ of $k$ by

$$
\Phi_{p}(k)=\frac{a_{0}}{p}+\frac{a_{1}}{p^{2}}+\frac{a_{2}}{p^{3}}+\cdots+\frac{a_{r}}{p^{r+1}} .
$$

If $p=2$, the sequence of $\Phi_{2}(k)$, for $k=0,1,2, \ldots$, is called the Van der Corput sequence [22].

Let $d$ be the dimension of the space to be sampled. Any sequence $p_{1}, p_{2}, \ldots, p_{d-1}$ of prime numbers defines a sequence $\Phi_{p_{1}}, \Phi_{p_{2}}, \ldots, \Phi_{p_{d-1}}$ of functions, whose corresponding $k$-th $d$-dimensional Hammersley point is

$$
\left(\frac{k}{n}, \Phi_{p_{1}}(k), \Phi_{p_{2}}(k), \ldots, \Phi_{p_{d-1}}(k)\right) \quad \text { for } k=0,1,2, \ldots, n-1 .
$$

where $p_{1}<p_{2}<\cdots<p_{d-1}$ and $n$ is the total number of Hammersley points. To evaluate the function $\Phi_{p}(k)$, the following algorithm can be used. 


$$
\begin{aligned}
& p^{\prime}=p, k^{\prime}=k, \Phi=0 \\
& \text { while } k^{\prime}>0 \text { do } \\
& a=k^{\prime} \bmod p \\
& \Phi=\Phi+\frac{a}{p^{\prime}} \\
& k^{\prime}=\operatorname{int}\left(\frac{k^{\prime}}{p}\right) \\
& p^{\prime}=p^{\prime} p
\end{aligned}
$$

where int $(x)$ returns the integer part of $x$.

The above algorithm has a complexity of $O\left(\log _{p} k\right)$ for evaluating the $k$-th point. Hence the worst case bound of the algorithm for generating $(N+1)$ points is,

$$
\begin{aligned}
& \log _{p}(1)+\log _{p}(2)+\cdots+\log _{p}(N-1)+\log _{p}(N) \\
\leq & \log _{p}(N)+\log _{p}(N)+\cdots+\log _{p}(N)+\log _{p}(N) \\
= & N \log _{p} N .
\end{aligned}
$$

A Pascal implementation of this algorithm can be found in [10]. In most computer graphics applications, the dimension of the sampled space is either 2 or 3 . In this paper, we concentrate on the generation of a uniformly distributed point set on the surface of 2D plane and sphere using Hammersley points. Higher dimensional sets can be similarly generated using formulæ (1-3).

Points on the 2D Plane On the 2D plane, formula (3) simplifies to

$$
\left(\frac{k}{n}, \Phi_{p_{1}}(k)\right) \text { for } k=0,1,2, \ldots, n-1 .
$$

The range of $\frac{k}{n}$ is $[0,1)$, while that of $\Phi_{p_{1}}(k)$ is $[0,1]$. For computer applications, a good choice of the prime $p_{1}$ is $p_{1}=2$. The evaluation of $\Phi_{2}(k)$ can be done efficiently with about $\log _{2}(k)$ bitwise shifts, multiplications and additions: no division is necessary. The $\mathrm{C}$ implementation of 2D Hammersley points with base 2 (Van der Corput sequence) is shown in the Appendix (source code 1). We shift $\frac{k}{n}$ by 0.5 to center the sequence. Otherwise, $\Phi_{p_{1}}(0)$ will always equal 0 for any $n$, which is a undesirable effect.

However, the original Hammersley algorithm is not hierarchical. This is due to the first coordinate $\frac{k}{n}$, which for different values of $n$ results in different sets of points. This can be resolved by using two $p$-adic Van der Corput sequences with different prime numbers $p_{1}$ and $p_{2}$. This hierarchical version is known as the Halton point set $[15,22]$.

$$
\left(\Phi_{p_{1}}(k), \Phi_{p_{2}}(k)\right) \text { for } k=0,1,2, \ldots, n-1 .
$$

Since both functions $\Phi_{p_{1}}(k)$ and $\Phi_{p_{2}}(k)$ are hierarchical (being independent of $n$ by construction), so are the Halton point sets. Source code 2 in the Appendix implements the Halton point sets on the 2D plane. 
Points on the Sphere To generate directional vectors, or (equivalently) points on the spherical surface, the following mappings [21] is needed:

$$
\left(\frac{k}{n}, \Phi_{p}(k)\right) \mapsto(\phi, t) \mapsto\left(\sqrt{1-t^{2}} \cos \phi, \sqrt{1-t^{2}} \sin \phi, t\right)^{T}
$$

The first, from $\left(\frac{k}{n}, \Phi_{p}(k)\right)$ to $(\phi, t)$, is simply a linear scaling to the required cylindrical domain, $(\phi, t) \in[0,2 \pi) \times[-1,1]$. The mapping from $(\phi, t)$ to $\left(\sqrt{1-t^{2}} \cos \phi, \sqrt{1-t^{2}} \sin \phi, t\right)^{T}$ is $z$-preserving radial projection from the unit cylinder $C=\left\{(x, y, z) \mid x^{2}+y^{2}=\right.$ $1|z| \leq 1\}$ to the unit sphere.

As before, the coordinate $\frac{k}{n}$ makes the scheme non-hierarchical. Halton points on the sphere can be generated in a similar manner by using two $p$-adic Van der Corput sequences with different prime bases.

$$
\left(\Phi_{p_{1}}(k), \Phi_{p_{2}}(k)\right) \mapsto(\phi, t) .
$$

Source code 3 in Appendix A shows the C implementation of Hammersley points on the sphere, with a similar 0.5-shift applied to prevent a fixed sample point from appearing at the South Pole, and source code 4 shows the Halton point version. For efficiency of computation, we fixed $p_{1}=2$ while leaving $p_{2}$ as a user input. This restriction can be trivially removed.

\section{Appearance}

Figures 2 and 3 show the Hammersley points with different bases, on the plane and the sphere respectively. We generated 500 samples for the planar test and 1000 for the sphere test. Figures 2(a) and 3(a) are the patterns of random sampling on the plane and the sphere respectively. Compared to the random sampling pattern (figure 2(a)), the Hammersley point set with $p_{1}=2$ (figure 2(b)) gives a pleasant, less clumped pattern. The points are uniformly distributed without a perceptible pattern. Among the patterns with different bases, Hammersley point set with $p_{1}=2$ (figure 2(b)) also gives the most uniformly distributed pattern. As the base $p_{1}$ increases (from figures 2(b) to 2(f) ), the pattern becomes more and more regular. The points tend to line up in slanting lines, which will clearly increase aliasing problems.

The same progression affects spherical sampling patterns (figures 3(b)-3(f)). When $p_{1}=2$, it gives the best uniformly distributed pattern on the sphere. Cui et al. [5] measures the uniformity of Hammersley points with $p_{1}=2$ on the sphere using the generalized discrepancy. It gives the lowest generalized discrepancy (most uniformly distributed) among the methods tested. As $p_{1}$ increases (from figures 3(b) to 3(f)), points start to line up and form regular lines on the sphere. The position of the pole (marked with an arrow) becomes distinguishable from the pattern.

The Halton point sets give patterns with varying uniformity and regularity (figures 4 and 5). To compare the effect of different bases $p_{1}$ and $p_{2}$, all patterns generated with $p_{1}=2$ are placed on the left, while those with $p_{1}=3$ are on the right. The omission of the case where $p_{1}=p_{2}=3$ is due to the constraint $p_{1}<p_{2}$. Figure 4(b) 
gives a pattern with somewhat aligned points. Others give rather pleasant appearances. Among the point sets tested, none give a better pattern than Hammersley points with $p_{1}=2$. In general the patterns of Halton points are quite unpredictable. Nevertheless, after transforming the points to the sphere, there is no way to distinguish the pole and equator of the sphere (figures 5(a) $-5(\mathrm{e})$ ). They are not as uniformly distributed as Hammersley point set with $p_{1}=2$, but there is no lining-up like that observed in Hammersley points.

\section{Ray Tracing Experiments}

The method is tested in a ray-tracer. Instead of generating a distinct sampling pattern for each pixel, a single sampling pattern is generated for the whole screen. Otherwise, the sampling pattern for each pixel will be the same, since the Hammersley and Halton points are actually deterministic. Hence, we can only specify the average sample per pixel.

Two scenes are chosen for testing: checker (figure 1(a)) and checker 45 (figure 1(b)). The "correct" images, used for calculating the pixel error $E$ in luminance, are produced by sampling the scenes using jittered sampling with 400 samples per pixel. Five other sampling schemes, jittered sampling, multi-jittered sampling, Poisson disk, random and regular sampling, are included for comparison. All of these five sampling schemes are tested with 16 samples per pixel, while the Hammersley and Halton point sets are tested with an average of 16 samples per pixel.

Four statistical data are recorded: average (Mean $|E|)$, standard deviation (S.D. $(|E|)$ ), root-mean-square (R.M.S.(E)) and maximum (Max.(|E|)) of the absolute pixel error in luminance. Tables 1 and 2 show the statistics from test scenes checker and checker 45 respectively. Methods listed in the tables are ranked by their performance.

Among tested methods, Hammersley point set with $p_{1}=2$ gives the lowest average, standard derivation and root-mean-square of absolute pixel errors in both test scenes. Multi-jittered sampling is the first runner-up. Hammersley point sets with higher bases $\left(p_{1}>3\right)$ are not tested due to the lining-up phenomenon, which certainly introduces aliasing. For Halton point sets, we arbitrarily choose two bases for testing, since there is no general trend in the appearance of the patterns. In our experiment, Hammersley point sets are better than the tested Halton point sets. Both Hammersley and Halton point sets give lower error than that of traditional jittered sampling and Poisson disk except the multi-jittered method.

\section{Conclusions}

The Hammersley point set with $p_{1}=2$ gives the most uniformly distributed sampling pattern. For higher $p_{1}$, the points tend to align and reduce its usefulness. Although the Halton point sets do not give patterns as uniformly distributed as Hammersley point sets, they do not have the line-up problem and it allows incremental sampling. 
Hammersley points and Halton points have been proved useful for quasi-Monte Carlo integration. The methods are applied to ray tracing applications with a significant improvement in pixel error. The complexity of both Hammersley and Halton points generation algorithms is $O\left(N \log _{p} N\right)$, which is smaller than that of Poisson disk.

\section{Acknowledgements}

We would like to thank Prof. Timothy Poston of National University of Singapore for his careful proofread and useful suggestions. We would also like to express our thanks to the editor and reviewers for their valuable advices and comments.

\section{References}

[1] James Case. Wall street's dalliance with number theory. SIAM News, pages $8-9$, December 1995.

[2] Kenneth Chiu, Peter Shirley, and Changyaw Wang. Multi-jittered sampling. In Graphics Gems IV, pages 370-374. AP Professional, 1994.

[3] Robert L. Cook, Thomas Porter, and Loren Carpenter. Distributed ray tracing. In Computer Graphics (SIGGRAPH '84 Proceedings), pages 137-145, July 1984.

[4] Robert A. Cross. Sampling patterns optimized for uniform distribution of edges. In Graphics Gems V, pages 359-363. AP Professional, 1995.

[5] Jianjun Cui and Willi Freeden. Equidistribution on the sphere. SIAM Journal on Scientific Computing, 18(2):595-609, March 1997.

[6] Joseph M. Cychosz. Efficient generation of sampling jitter using look-up tables. In Graphics Gems, pages 64-74. AP Professional, 1990.

[7] D. P. Dobkin and D. Eppstein. Computing the discrepancy. In Proceedings of the 9th ACM Symposium on Computational Geometry, pages 47-52, 1993.

[8] D. P. Dobkin, David Eppstein, and Don P. Mitchell. Computing the discrepancy with applications to supersampling patterns. ACM Transactions on Graphics, 15(4):354-376, October 1996.

[9] D. P. Dobkin and D. P. Mitchell. Random-edge discrepancy of supersampling patterns. In Graphics Interface, pages 62-69, 1993.

[10] J. H. Halton and G. B. Smith. Radical-inverse quasi-random point sequence. Communications of the ACM, 7(12):701-702, December 1964.

[11] Stefan Heinrich and Alexander Keller. Quasi-monte carlo methods in computer graphics, part i: The qme buffer. Technical report, University of Kaiserslautern, 1994. 242/94.

[12] Stefan Heinrich and Alexander Keller. Quasi-monte carlo methods in computer graphics, part ii: The radiance equation. Technical report, University of Kaiserslautern, 1994. 243/94.

[13] Alexander Keller. A quasi-monte carlo algorithm for the global illumination problem in the radiosity setting. In Proceedings of Monte Carlo and Quasi-Monte Carlo Methods in Scientific Computing, pages 239-251. Springer-Verlag, June 1995. 
[14] H. Niederreiter. Quasirandom sampling computer graphics. In Proceedings of the 3rd International Seminar on Digital Image Processing in Medicine, pages 29-33, 1992.

[15] H. Niederreiter. Random Number Generation and Quasi-Monte Carlo Methods. CBMSNSF, SIAM, Philadelphia, 1992.

[16] Ryutarou Ohbuchi and Masaki Aono. Quasi-monte carlo rendering with adaptive sampling. Technical report, Tokyo Research Laboratory, IBM Japan Ltd., 1996.

[17] S. H. Paskov and J. F. Traub. Faster valuing of financial derivatives. Journal of Portfolio Management, 22:113-120, 1995.

[18] Peter Shirley. Discrepancy as a quality measure for sample distributions. In Proceedings of Eurographics, pages 183-193, 1991.

[19] Peter Shirley. Physically Based Lighting Calculations for Computer Graphics. PhD thesis, University of Illinois at Urbana-Champaign, 1991.

[20] Ken Shoemake. Interval sampling. In Graphics Gems II, pages 394-395. AP Professional, 1991.

[21] Jerome Spanier and Ely M. Gelbard. Monte Carlo Principles and Neutron Transport Problems. Addison-Wesley, New York, N.Y., 1969.

[22] Shu Tezuka. Uniform Random Numbers: Theory and Practice. Kluwer Academic Publishers, 1995.

[23] Joseph Traub. In math we trust. What's Happening in the Mathematical Sciences, 3:101-111, 1996. 


\section{Web Information:}

All source codes in the appendix and a demostration program showing the appearances of various Hammersley and Halton point sets are available at

http://www.acm.org/jgt/papers/WongLukHeng 97

Tien-Tsin Wong, Dept. of Computer Science \& Eng., The Chinese University of Hong Kong, Shatin, Hong Kong.

email: ttwongeacm.org

web: http://www.cse.cuhk.edu.hk/ ttwong/

Wai-Shing Luk, Departement Computerwetenschappen, Katholieke Universiteit Leuven, Celestijnenlaan 200A, B-3001 Heverlee, Belgium.

email: Wai-Shing.Luk@cs.kuleuven.ac.be

web: http://www.cs.kuleuven.ac.be/ dannyl/

Pheng-Ann Heng, Dept. of Computer Science \& Eng., The Chinese University of Hong Kong, Shatin, Hong Kong.

email: pheng@cse.cuhk.edu.hk

web: http://www.cse.cuhk.edu.hk/ pheng/ 


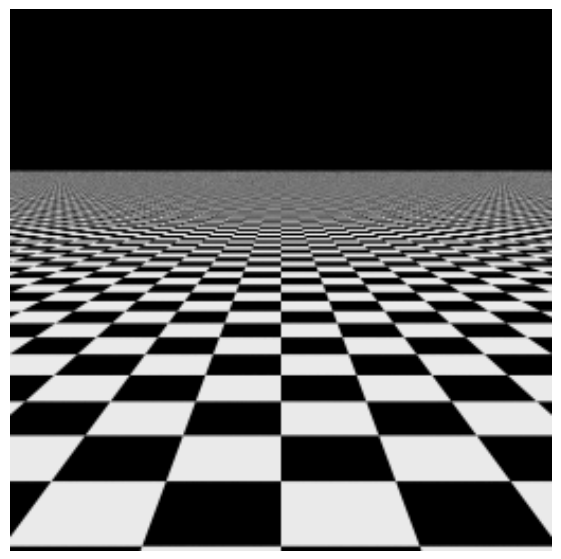

(a)checker

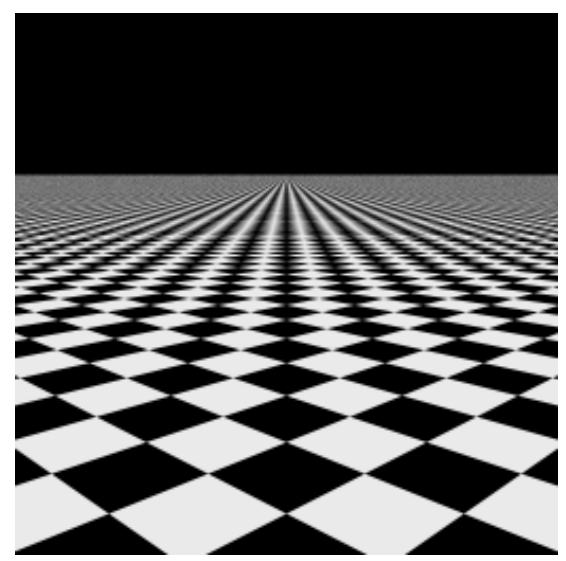

(b)checker 45

Figure 1: The two test scenes used in the sampling test.

\begin{tabular}{|l|c|c|c|c|}
\hline Methods & Mean $(|E|)$ & S.D. $(|E|)$ & R.M.S.(E) & Max.( $|E|)$ \\
\hline Hamm., $p_{1}=2$ & 0.0086 & 0.0247 & 0.0261 & 0.3451 \\
multi-jitter, $n=4, N=16$ & 0.0091 & 0.0261 & 0.0277 & 0.3843 \\
Hamm., $p_{1}=3$ & 0.0097 & 0.0265 & 0.0282 & 0.3961 \\
Halton, $p_{1}=2, p_{2}=7$ & 0.0105 & 0.0280 & 0.0299 & 0.3451 \\
Halton, $p_{1}=2, p_{2}=3$ & 0.0110 & 0.0291 & 0.0312 & 0.3686 \\
jittered, $4 \times 4$ & 0.0128 & 0.0335 & 0.0358 & 0.3804 \\
Poisson, $d=0.2$ & 0.0132 & 0.0338 & 0.0363 & 0.3804 \\
random & 0.0179 & 0.0443 & 0.0478 & 0.3961 \\
regular & 0.0188 & 0.0491 & 0.0526 & 0.5098 \\
\hline
\end{tabular}

Table 1: Statistics of ray traced image checker. $E$ is the pixel error in luminance.

\begin{tabular}{|l|c|c|c|c|}
\hline Methods & Mean $(|E|)$ & S.D. $(|E|)$ & R.M.S.(E) & Max. $(|E|)$ \\
\hline Hamm., $p_{1}=2$ & 0.0101 & 0.0264 & 0.0282 & 0.3882 \\
multi-jitter, $n=4, N=16$ & 0.0103 & 0.0270 & 0.0289 & 0.3686 \\
Hamm., $p_{1}=3$ & 0.0106 & 0.0274 & 0.0294 & 0.4431 \\
Halton, $p_{1}=2, p_{2}=3$ & 0.0114 & 0.0287 & 0.0309 & 0.3882 \\
Halton, $p_{1}=2, p_{2}=7$ & 0.0131 & 0.0289 & 0.0310 & 0.4118 \\
jittered, $4 \times 4$ & 0.0131 & 0.0332 & 0.0357 & 0.3765 \\
Poisson, $d=0.2$ & 0.0133 & 0.0332 & 0.0358 & 0.4118 \\
regular & 0.0138 & 0.0393 & 0.0416 & 0.5059 \\
random & 0.0185 & 0.0446 & 0.0483 & 0.4000 \\
\hline
\end{tabular}

Table 2: Statistics of ray traced image checker 45. $E$ is the pixel error in luminance. 


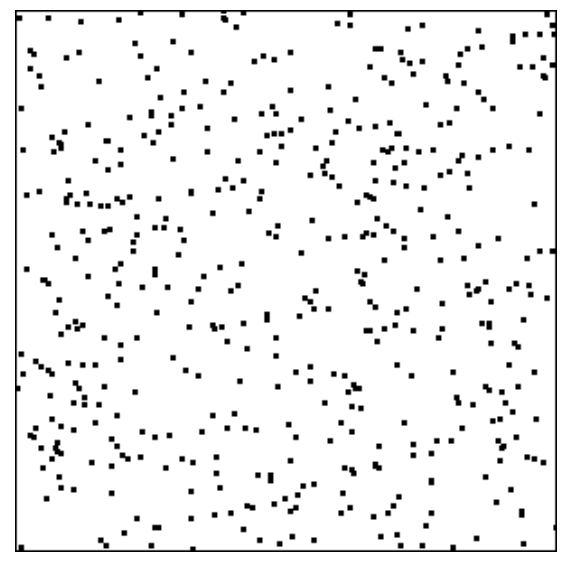

(a) random

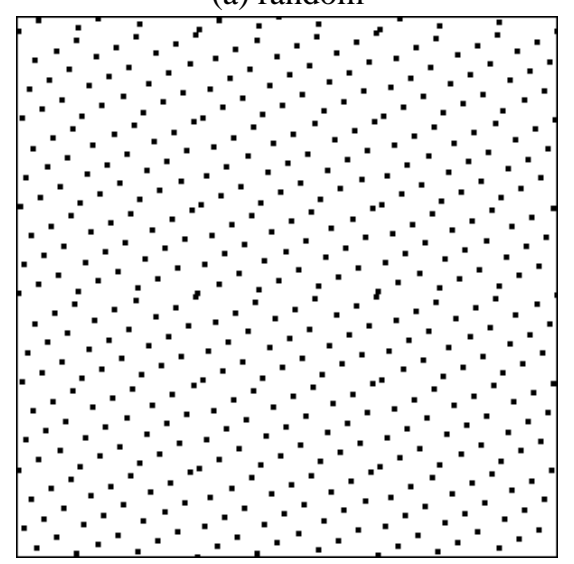

(c) $p_{1}=3$

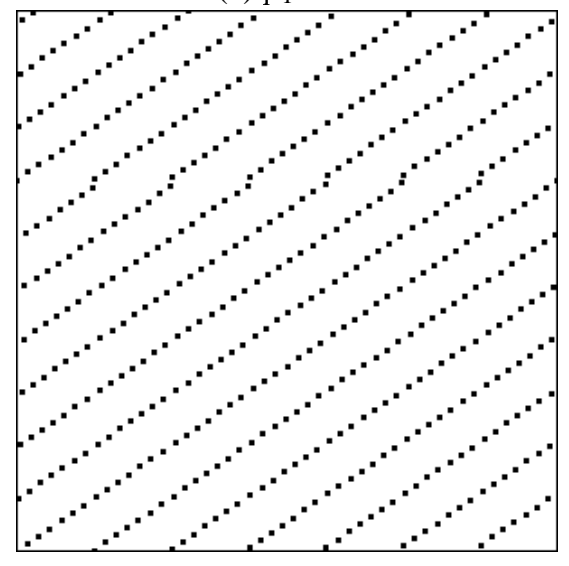

(e) $p_{1}=7$

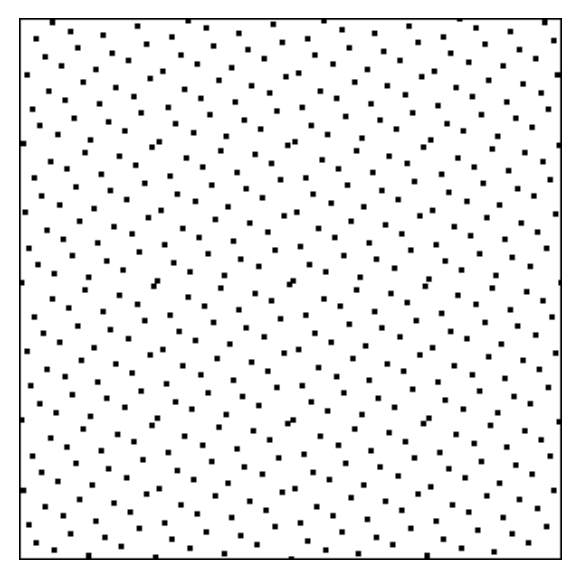

(b) $p_{1}=2$

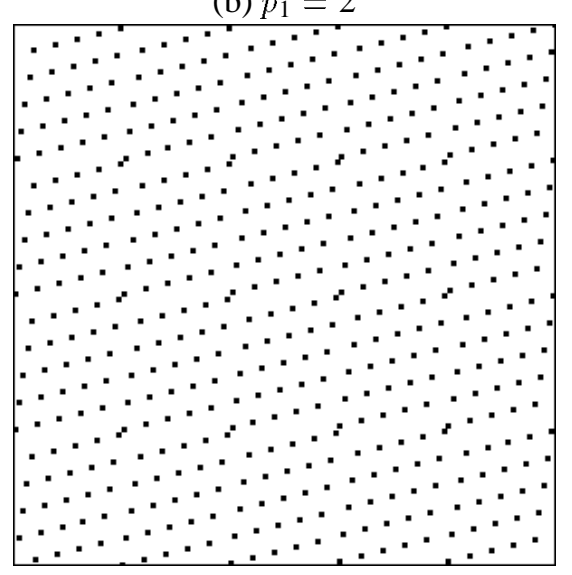

(d) $p_{1}=5$

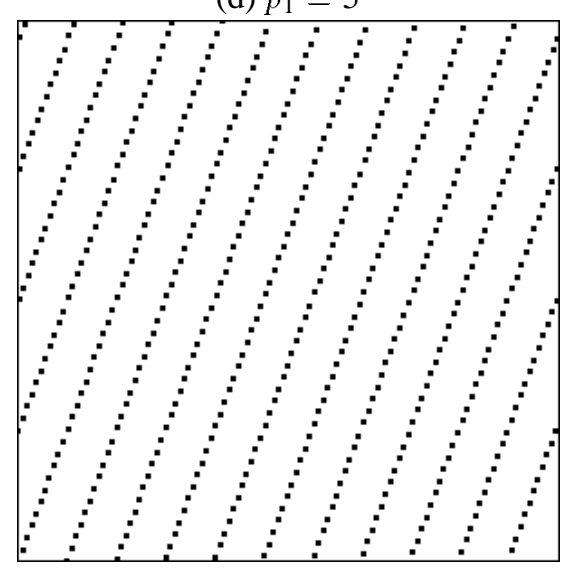

(f) $p_{1}=11$

Figure 2: Hammersley points on the 2D plane $(n=500)$. 


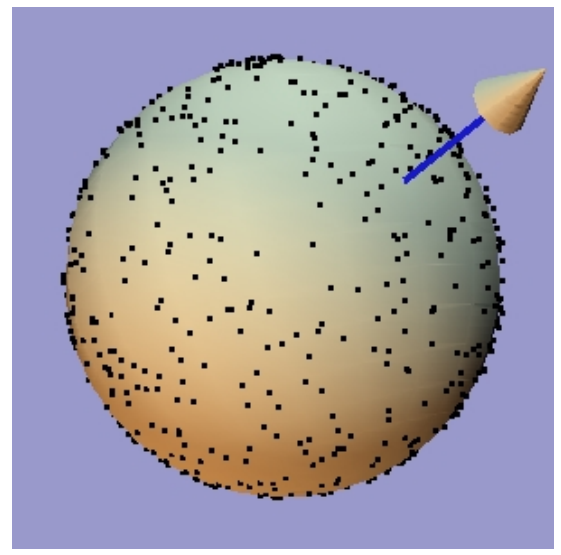

(a) random

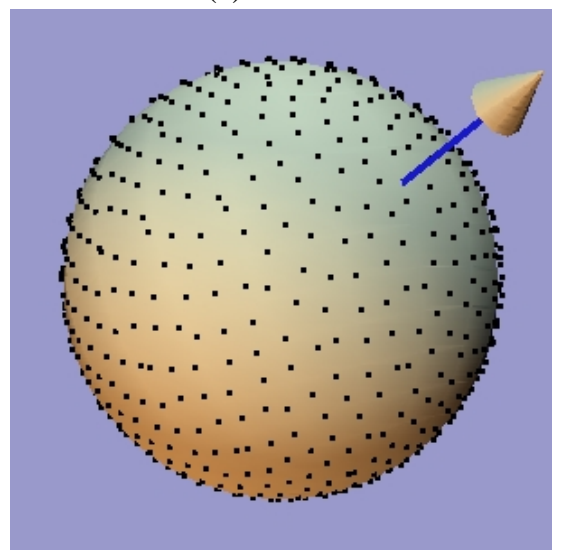

(c) $p_{1}=3$

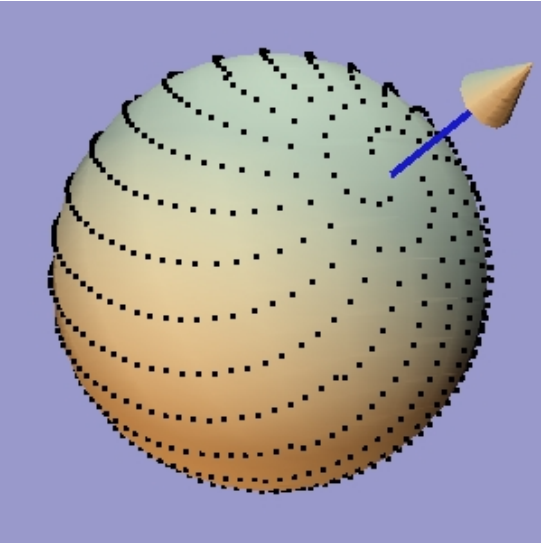

(e) $p_{1}=7$

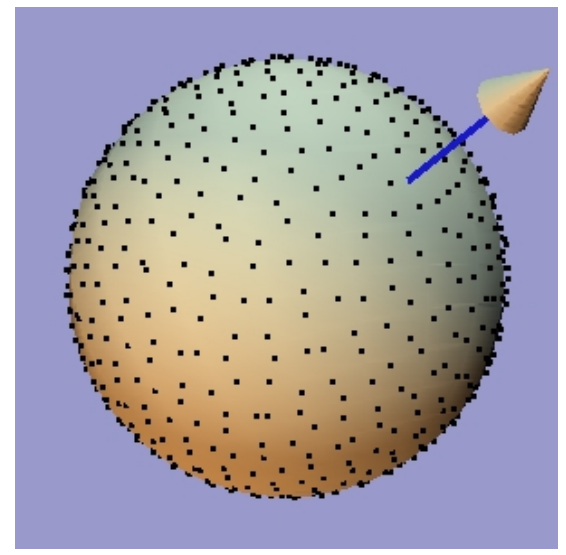

(b) $p_{1}=2$

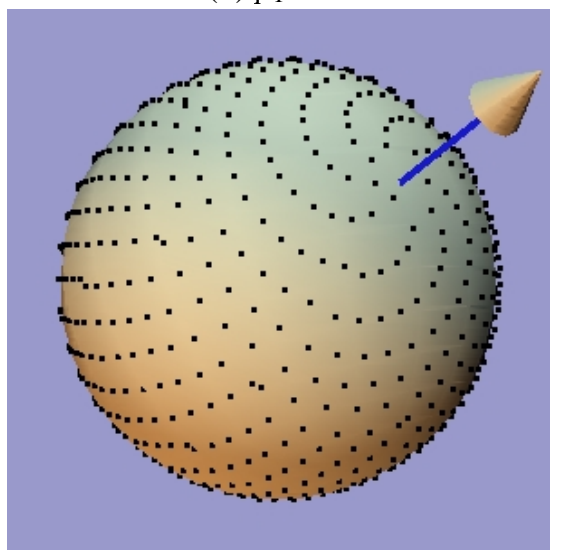

(d) $p_{1}=5$

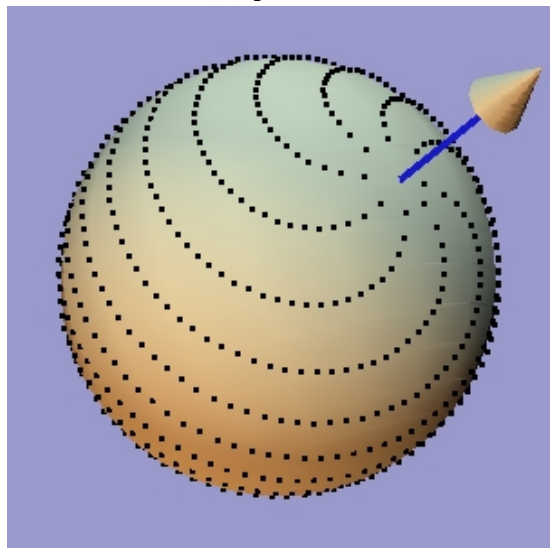

(f) $p_{1}=11$

Figure 3: Hammersley points on the sphere $(n=1000)$. 


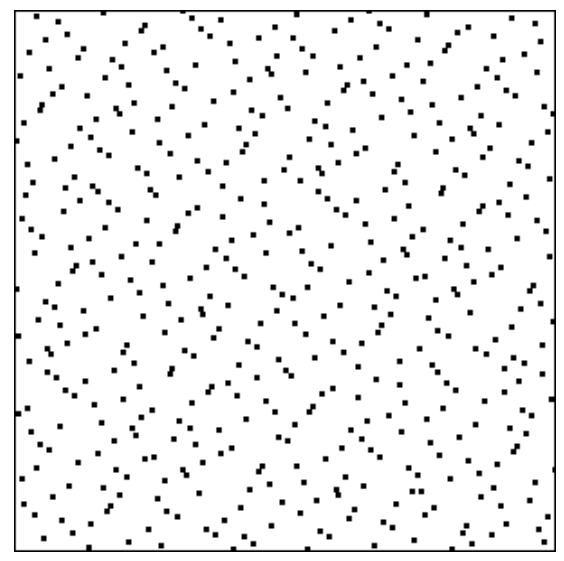

(a) $p_{1}=2, p_{2}=3$

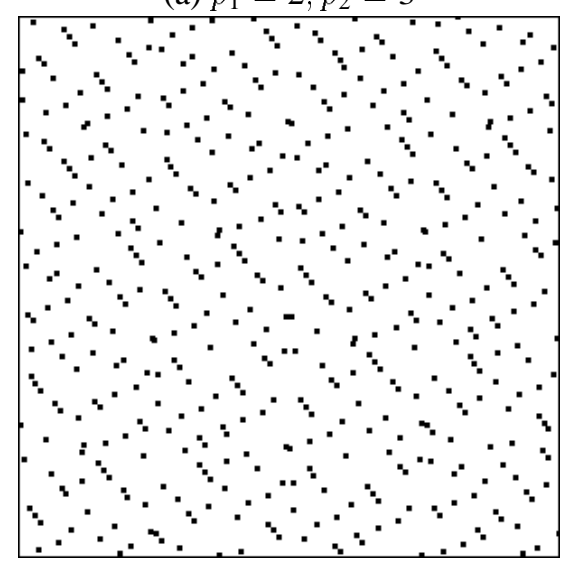

(b) $p_{1}=2, p_{2}=5$

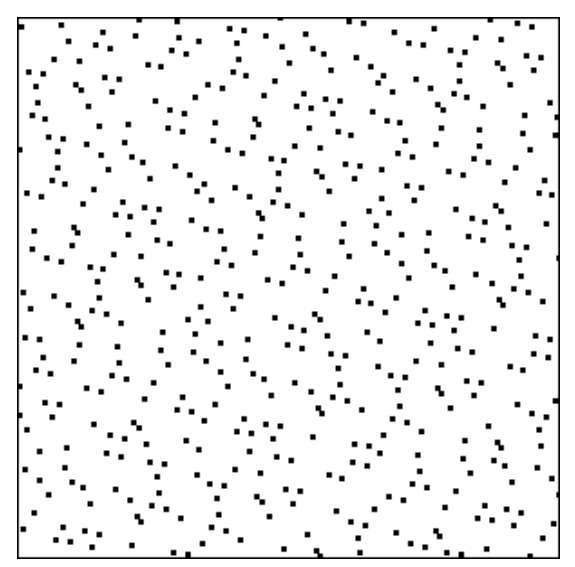

(d) $p_{1}=2, p_{2}=7$

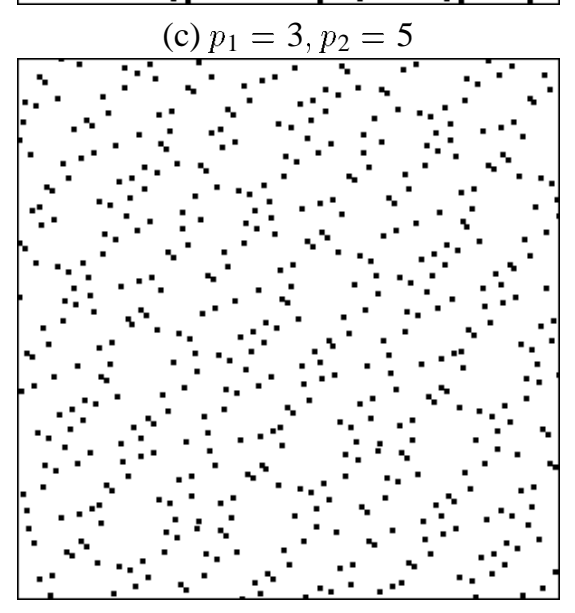

(e) $p_{1}=3, p_{2}=7$

Figure 4: Halton points with different bases on the 2D plane $(n=500)$. 


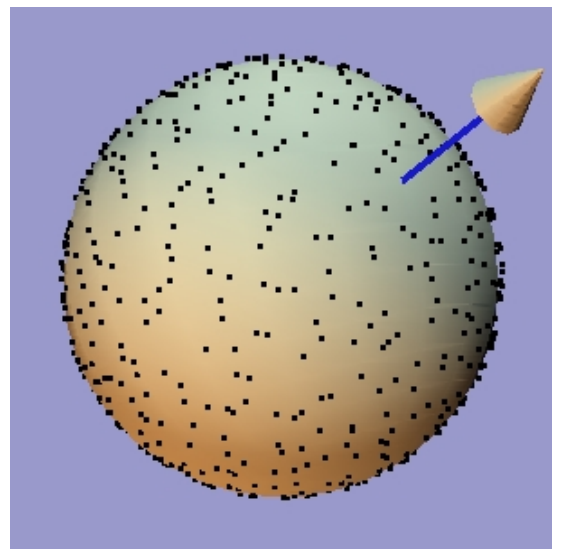

(a) $p_{1}=2, p_{2}=3$
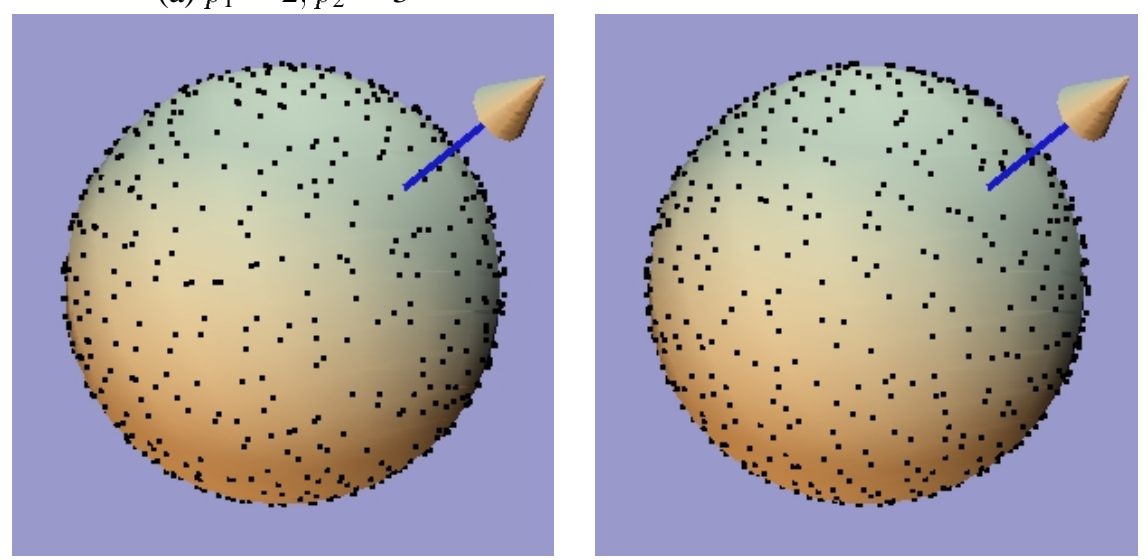

(b) $p_{1}=2, p_{2}=5$

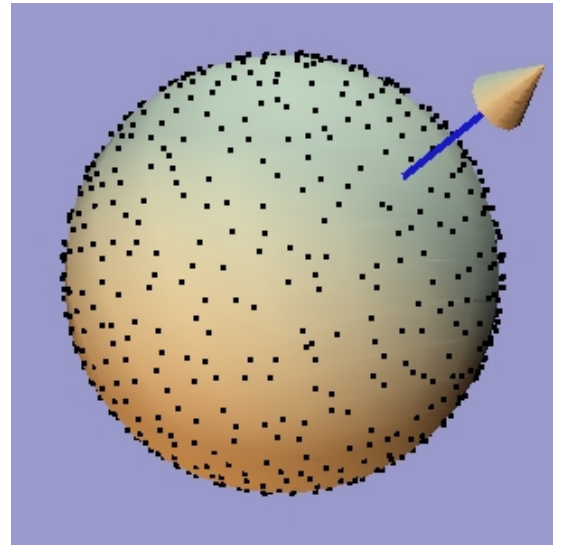

(d) $p_{1}=2, p_{2}=7$

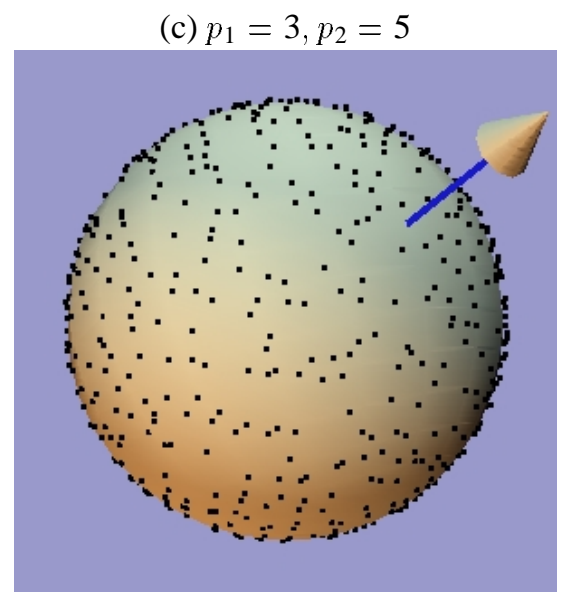

(e) $p_{1}=3, p_{2}=7$

Figure 5: Halton points with different bases on the sphere $(n=1000)$. 


\section{A Appendix: Source Code}

Source Code 1 Hammersley Points on 2 D Plane with $p_{1}=2$

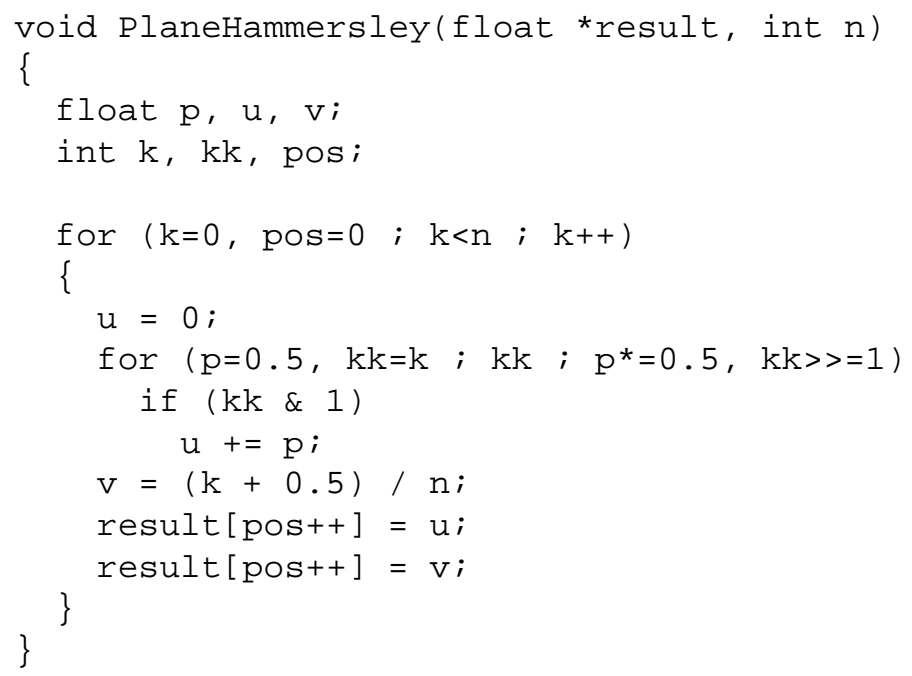

Source Code 2 Halton Points on $2 D$ Plane with $p_{1}=2$

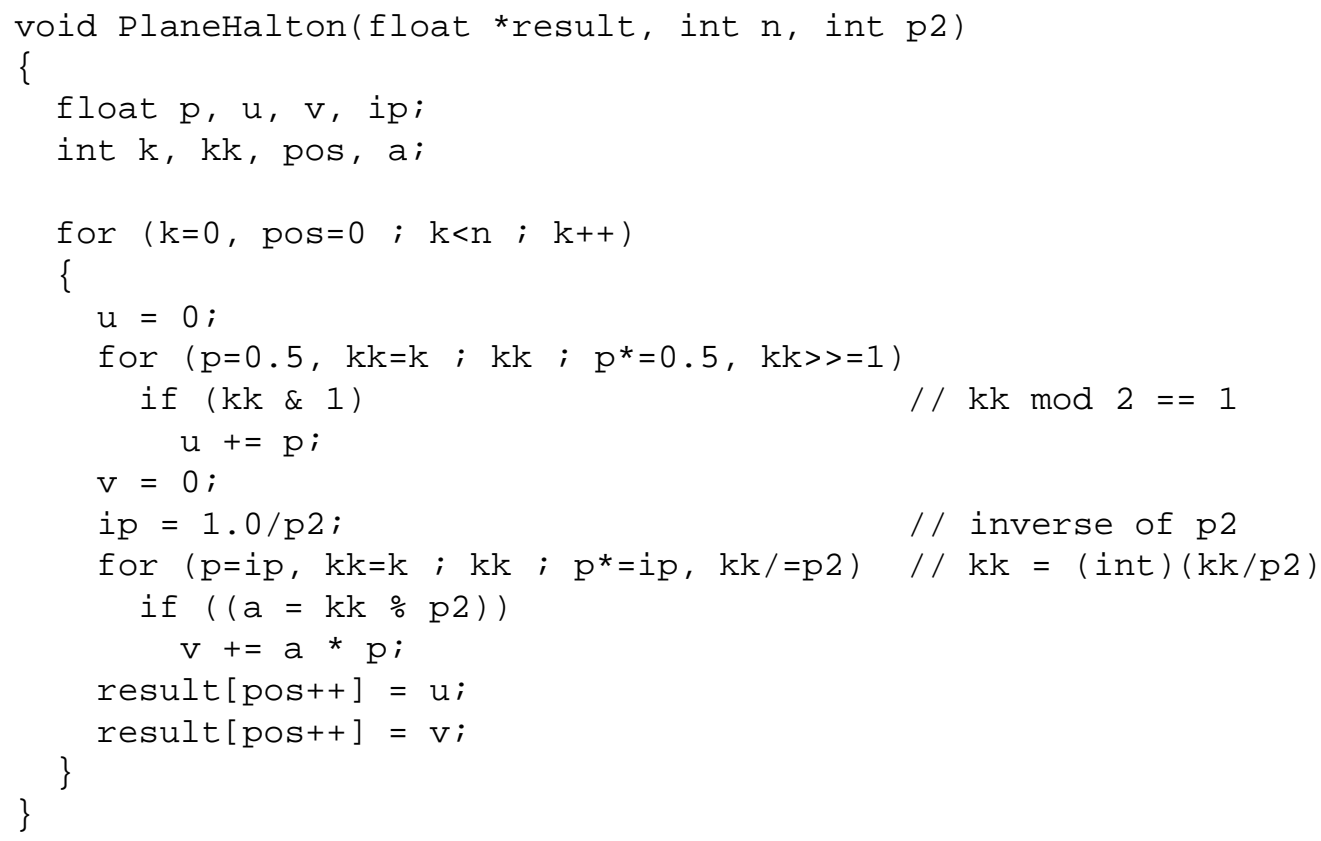


Source Code 3 Hammersley Points on Sphere with $p_{1}=2$

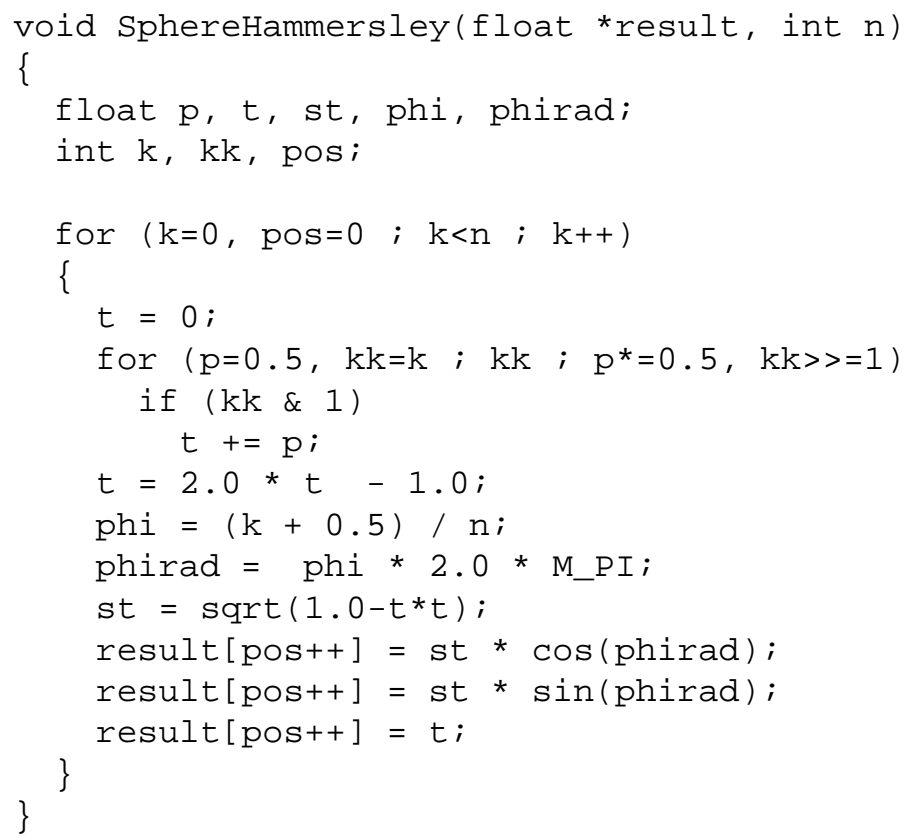

Source Code 4 Halton Points on Sphere with $p_{1}=2$

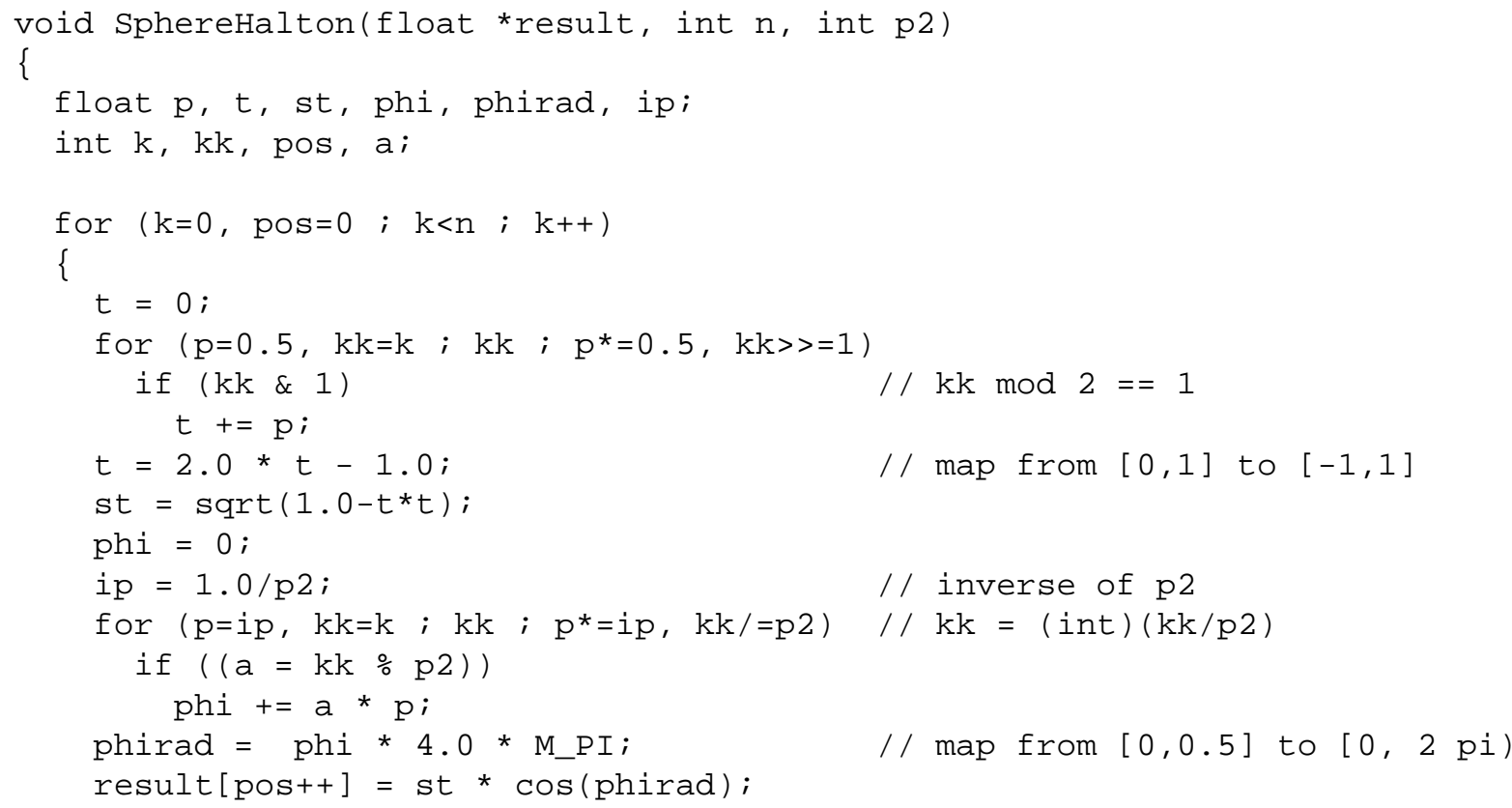


result [post+] = st * $\sin ($ phirad);

\}

result $[$ post+] $=t$;

\} 